\title{
Is Ultrasound Effective in Diagnosing Internal Derangements of the Knee?
}

\section{S. K. Venkatesh Gupta, Sugnanam Sirish Aditya}

Department of Orthopaedics, Mamata Medical College/General Hospital, Khammam, India. Email: sirish.sunny@gmail.co

Received October $30^{\text {th }}$, 2013; revised November $29^{\text {th }}$, 2013; accepted December $15^{\text {th }}, 2013$

Copyright (c) 2013 S. K. Venkatesh Gupta, Sugnanam Sirish Aditya. This is an open access article distributed under the Creative Commons Attribution License, which permits unrestricted use, distribution, and reproduction in any medium, provided the original work is properly cited. In accordance of the Creative Commons Attribution License all Copyrights (C) 2013 are reserved for SCIRP and the owner of the intellectual property S. K. Venkatesh Gupta, Sugnanam Sirish Aditya. All Copyright (C) 2013 are guarded by law and by SCIRP as a guardian.

\begin{abstract}
Internal derangement of knee significantly affects daily activities of patients and management of such cases with accurate diagnosis and early treatment is of utmost importance. This study determines the benefits of arthroscopy directly and also compares the sensitivity and specificity of ultrasound, MRI and arthroscopic findings in diagnosing internal derangements of the knee. This is a prospective study of 50 cases that includes patients having knee pain and instability of joint for more than 6 weeks, symptoms of locking of knee joint or effusion and having no bony injury as confirmed by $\mathrm{X}$ ray. Results were analysed and sensitivity and specificity were calculated. The present study supports that the clinical diagnosis is of primary necessity, while ultrasound and MRI are additional diagnostic tools in diagnosing IDK. Arthroscopy combines more accurate diagnosing tool and therapeutic modality, which is a more convenient, economical and convincing technique to both surgeon and patient alike. Although ultrasound is less accurate than MRI, it is cost effective and available at most of the peripheral centres. So it is better to do ultrasound rather than MRI for diagnosing IDK in peripheral centres and refer to specialty clinics or tertiary centres for further diagnosis and treatment.
\end{abstract}

Keywords: Knee; Menisci; MRI; Ultrasound; ACL; PCL; Arthroscopy

\section{Introduction}

The modern high speed motor vehicular trauma and the sporting life style have become obsessions in all age groups, causing damage to bone and soft tissue elements especially of knee and causing internal derangement injuries. They account for a large number of referred cases to specialty clinics and referral centers not only from the peripherals and general practitioners but also from other major centres. As it comes in the way of daily activities and significantly affects financial earning of the person and family, it is important to deal such cases with accurate diagnosis and early treatment [1-4].

Combined lesions are more difficult to diagnose. The clinical examination and special tests to determine instability and internal derangement still stand as preliminary and gold standards, more reliable and cost effective ways of diagnosing such knee problems. Literature reviews show that clinical examination is not always accurate to diagnose IDK $[5,6]$. Ultrasound and MRI are non inva- sive tools in investigating internal derangements of knee, but false and misleading results are equally reported in the literature. The difficulty in diagnosing lesions of the knee is that different lesions in the knee joint can produce similar findings. Arthroscopy has been considered as the gold standard for the diagnosis of IDK, but is invasive, expensive and requires day surgery admission. Hence, this study is intended to determine the benefits of arthroscopy directly and also to compare the sensitivity and specificity of ultrasound, MRI and arthroscopic findings in diagnosing internal derangements of the knee.

\section{Methodology}

This is a prospective study of 50 cases admitted to Mamata General and Super Speciality Hospital between May 2011 and Dec 2012 having knee joint problem like pain and instability of knee joint for more than 6 weeks, those with recent symptoms of locking of knee joint or effusion and those with chronic knee pain having no 
bony injury confirmed by x ray were included. All patients were clinically examined and were evaluated with $\mathrm{x}$-ray, ultrasound, MRI scan and diagnostic arthroscopy. Patients treated for tuberculosis of knee and septic arthritis, chronic osteoarthrosis or ankylosis of knee and those who underwent arthroscopy previously were excluded from the study. A standard 7.5 $\mathrm{MHz}$ ultrasound probe and 0,3 Tesla MRI scanner was used in this study.

\subsection{Positioning during Ultrasound Examination}

Ultrasound of the knee was performed with a using a linear array $7.5 \mathrm{MHz}$ probe. The patient was initially placed supine with the knee extended. The anterior horns were examined from the medial and lateral aspects respectively. The knee was then flexed to 90 degrees and the probe rotated laterally to examine the Anterior Cruciate Ligament (ACL). This was a dynamic examination with the knee being serially extended during the procedure.

The patient was then turned prone and the posterior horns were examined from the medial and lateral aspects respectively. The Posterior Cruciate Ligament (PCL) was then examined with the probe rotated medially.

\subsection{Positioning of Patient during Arthroscopy}

Arthroscopy of knee is done by hanging limb by side of table with knee in $90^{\circ}$ degrees of flexion and for full range of motion at knee joint during procedure (Figure 1).

\subsection{Portal Placement}

In our complete study we used anteromedial and antero lateral portals for diagnostic arthroscopy (Figure 1).

\section{Results}

The study had 50 patients of which $40(80 \%)$ were males and $10(20 \%)$ were females.

In our study patients are in between 18 to 50 years of age.

More no of patients are in between 21 to 30 years of age (Chart 1).

Mean age of the study group was 28.3 years with age range between 19 - 43 years.

In our study of 50 patients there are different modes of injury in which Sports injury (64\%) was the most commonest cause of modality of injury followed by road traffic accident (16\%) (Chart 2).

All patients were clinically examined and were evaluated with X-ray, ultrasound, MRI scan and diagnostic arthroscopy.

$\mathrm{X}$ rays were done to rule out associated fractures.

In our study we screened four structures like lateral
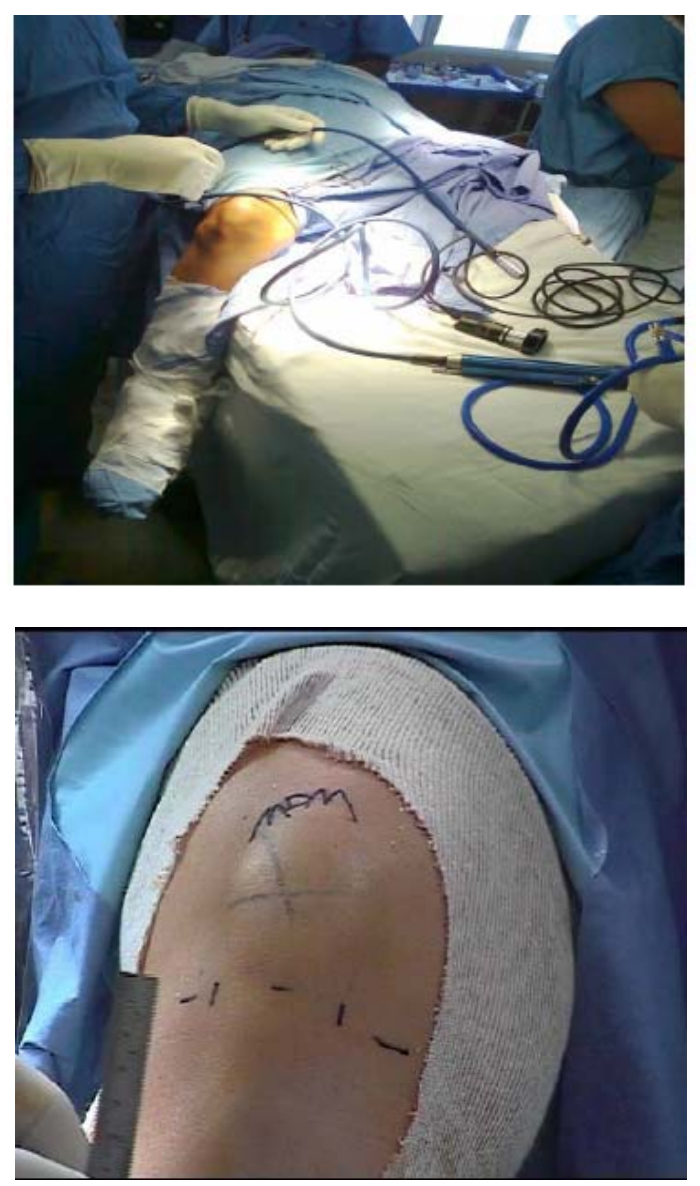

Figure 1. Patient positioning and portal placement in arthroscopy.

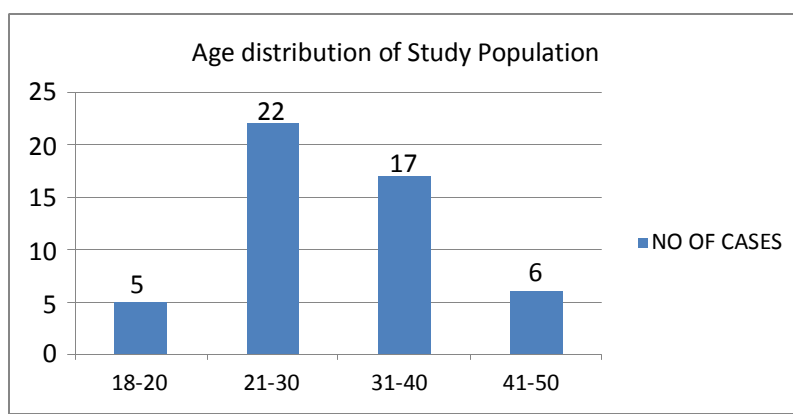

Chart 1. Age distribution of study population.

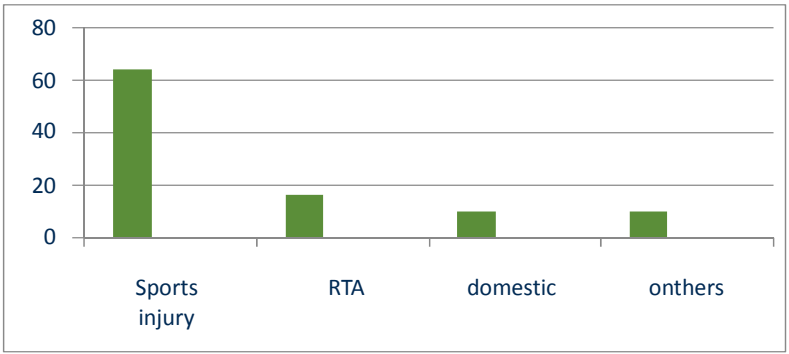

Chart 2. Graph deipicting mode of injury in patients with Internal derangement of knee in our study. 
meniscus, medial meniscus, anterior cruciate ligament and posterior cruciate ligament.

We compared results of ultrasound and MRI with diagnostic arthroscopy.

The results of 50 cases evaluated for internal derangement of knee with MRI, Ultrasound and arthroscopy are tabulated in Table $\mathbf{1 .}$

MRI had $74.1 \%$ sensitivity and $100 \%$ specificity in diagnosing ACL injuries while ultrasound scan had $74.2 \%$ sensitivity and $100 \%$ specificity in our study group. MRI had $96.1 \%$ sensitivity and $95.8 \%$ specificity in diagnosing Medial mensical injuries while ultrasound scan had $85.2 \%$ sensitivity and $86.1 \%$ specificity. MRI had $85.7 \%$ sensitivity and $100 \%$ specificity in diagnosing lateral meniscal injuries while ultrasound scan had 71.4\% sensitivity and $100 \%$ specificity. Our results show that ultrasound scan is equally good in picking up internal derangement of knee as compared to MRI scan (Table 2).

\section{Discussion}

Ultrasound diagnosis of orthopedic conditions has gathered pace in recent years. It has become popular because it is safe, quick, inexpensive and fairly reliable $[7,8]$. Ultrasound diagnosis of Internal derangement of knee has been tried in various studies with variable results. Most of these studies compare ultrasound with arthroscopy or arthrography [9-12]. Some of the cadaver and clinical studies on the diagnostic efficiency of ultrasound in Internal derangement of knee report high yield rates with sensitivity for menisci ranging from $76 \%$ to $100 \%$ and specificity from $50 \%$ to $97 \%[8,9,12]$. In other studies the sensitivity for menisci was as low as $30 \%$ to $40 \%$ [8,11]. The use of $7.5 \mathrm{MHz}$ probe for the visualization of the menisci is well established and our experience was the same (2). In our study the sensitivity and specificity for the lateral meniscus was $71.4 \%$ and $100 \%$ respectively. The sensitivity and specificity for the medial meniscus was $85.2 \%$ and $86.1 \%$ respectively. The number of lateral menisci was very low and therefore the results should be interpreted with caution. However, the number of medial menisci was significant so both MRI and ultrasound showed a high sensitivity and specificity. We also had good sensitivity and specificity for the ACL but did not have any PCL injuries in the series. All structures, i.e. medial meniscus, lateral meniscus, ACL and PCL were visualized clearly in all knees. Although there were no PCL injuries, the PCL was clearly visualized with ultrasound, whereas its visualization can be a problem on MRI. As it is shown from our results, the ultrasound findings in IDK compare well with both arthroscopy and MRI. In some cases, as for example the PCL, we think it was even more helpful than MRI. Ultrasound is not widely used as a diagnostic test for knee injuries, and there has to be a learning curve for its routine use $[11,13]$. Competent and experienced radiologist can diagnose IDK with help of ultrasound rather than expensive MRI. Ultrasound is available in all peripheral centres, where as MRI is only available in tertiary centres. So it is better to

Table 1. Summary of results.

\begin{tabular}{|c|c|c|c|c|c|c|c|c|c|}
\hline \multirow[t]{2}{*}{ Lesion } & \multicolumn{3}{|c|}{ Arthroscopy } & \multicolumn{3}{|c|}{ MRI } & \multicolumn{3}{|c|}{ ultrasound } \\
\hline & Torn & Normal & & Abnormal & Torn & Normal & Abnormal & Torn & Normal \\
\hline LM & 7 & 43 & & 6 & 0 & 44 & 0 & 5 & 45 \\
\hline \multirow[t]{2}{*}{ MM } & 27 & 23 & & 20 & 5 & 25 & 7 & 23 & 20 \\
\hline & Complete & Partial & Normal & & & & & & \\
\hline ACL & 20 & 7 & 23 & 13 & 7 & 30 & 3 & 17 & 30 \\
\hline PCL & 0 & 0 & 50 & 0 & 0 & 50 & 0 & 0 & 50 \\
\hline
\end{tabular}

LM - Lateral meniscus; MM - Medial meniscus; ACL- Anterior cruciate ligament PCL - Posterior cruciate ligament.

Table 2. Sensitivity \& specificity of MRI and ultrasound.

\begin{tabular}{ccccc}
\hline LESION & \multicolumn{2}{c}{ MRI } & \multicolumn{2}{c}{ ULTRASOUND } \\
\hline & SENSITIVITY & SPECIFICITY & SENSITIVITY & SPECIFICITY \\
\hline Lateral meniscus & $85.7 \%$ & 100 & $71.4 \%$ & $100 \%$ \\
Medial meniscus & $96.1 \%$ & $95.8 \%$ & $85.2 \%$ & $86.1 \%$ \\
Anterior cruciate ligament & $74.1 \%$ & 100 & $74.2 \%$ & $100 \%$ \\
Posterior cruciate ligament & N/A & N/A & N/A & N/A \\
\hline
\end{tabular}


do ultrasound rather than MRI for diagnosing IDK in peripheral centres and refer to specialty clinics or tertiary centres for further diagnosis and treatment [10-12].

\section{Conclusion}

The present study supports that the clinical diagnosis is of primary necessity. Ultrasound and MRI are additional diagnostic tools for diagnosing internal derangement of knee. Arthroscopy combines more accurate diagnosing tool and therapeutic modality, which is a more convenient, economical and convincing technique to both surgeon and patient alike. However, high expectations from patients knowing the diagnosis before undergoing interventional procedures like arthroscopy make ultrasound and MRI the highly demanding and needed technique in diagnosing IDK. MRI is considered as a more sensitive technique compared to ultrasound. However, MRI has false and misleading results as high as $20 \%-30 \%$ in knee pathologies as being reported in standard literature which is also confirmed in our study. Although ultrasound is less accurate than MRI, it is cost effective and available at most of the peripheral centres. We recommend that ultrasound be used at present as a screening test before a MRI is performed or where clinical examination is difficult or unclear.

\section{REFERENCES}

[1] H. Gerngross and C. Sohn, "Ultrasound Scanning for the Diagnosis of Meniscal Lesions of the Knee Joint," Arthroscopy, Vol. 8, No. 1, 1992, pp. 105-110. http://dx.doi.org/10.1016/0749-8063(92)90143-Y

[2] J. Grifka, J. Richter and M Gumtau, "Clinical and Sonographic Meniscus Diagnosis,” Orthopäde, Vol. 23, No. 2, 1994, pp. 102-111.

[3] F. Corbetti and G. Tomasella, "Meniscal Injuries of the Knee-Arthrographic and Echographic Study,” Radiologia Medica, Vol. 77, No. 3, 1989, pp. 187-194.
[4] M. Fusting and H. R. Casser, "Dynamic Examination Technique in Meniscus Sonography," Sportverletz Sportschaden, Vol. 5, No. 1, 1991, pp. 27-36.

[5] H. Gerngross and C. Sohn, "Ultrasound Scanning for the Diagnosis of Meniscal Lesions of the Knee Joint," Arthroscopy, Vol. 8, No. 1, 1992, pp. 105-110. http://dx.doi.org/10.1016/0749-8063(92)90143-Y

[6] J. Grifka, J. Richter and M. Gumtau, "Clinical and Sonographic Meniscus Diagnosis,” Orthopäde, Vol. 23, No. 2, 1994, pp. 102-111.

[7] T. Heuchemer, G. Bauer, J. M. Friedrich and G. Bargon, “Clinical Use of Meniscus Sonography,” Bildgebung, Vol. 56, No. 3, 1987-1989, pp. 118-123.

[8] J. Jerosch, W. H. Castro, H. U. Sons and W. Winkelmann, "The Value of Sonography in Injuries of the Knee Joint," Ultraschall in der Medizin, Vol. 10, No. 5, 1998, pp. 275281. http://dx.doi.org/10.1055/s-2007-1006007

[9] J. Mattli, P. Holzach and P. Soklic, "Meniscus Ultrasound -A Reliable Way for the Diagnosis of Meniscus Lesions?” Z Unfallchir Versicherungsmed, No. 1, 1993, pp. $133-140$.

[10] J. Richter, J. Grifka, A. Fissler-Eckhoff, et al., "Ultrasound Morphologic Criteria in Evaluating Meniscus ChangesAn Experimental Study," Z Orthop Ihre Grenzeb, Vol. 134, No. 2, 1996, pp. 137-143. http://dx.doi.org/10.1055/s-2008-1039785

[11] C. Sohn, H. Gerngross, W. Bahren and W. Swobodnik, "Sonography of the Meniscus and Its Lesions," Ultraschall in der Medizin, Vol. 8, No. 1, 1987, pp. 32-36. http://dx.doi.org/10.1055/s-2007-1011654

[12] C. Sohn, H. Gerngross, W. Bahren and B. Danz, "Meniscus Sonography-Alternative to Invasive Meniscus Diagnosis?” Deutsche Medizinische Wochenschrift, Vol. 112, No. 15, 1987, pp. 581-584. http://dx.doi.org/10.1055/s-2008-1068101

[13] C. Sohn, H. R. Casser and W. Swobodnik, "Ultrasound Criteria of a Meniscus Lesion," Ultraschall in der Medizin, Vol. 11, No. 2, 1990, pp. 86-90. 